\title{
Income inequality measures and the middle class
}

\author{
Oleg I. Pavlov ${ }^{1, *}$, and Olga Yu. Pavlova ${ }^{2}$ \\ ${ }^{1}$ Peoples' Friendship University of Russia (RUDN University), Miklukho-Maklaya Street, 6, 117198 \\ Moscow, Russian Federation \\ ${ }^{2}$ All-Russian correspondence multidisciplinary school, B-234 Vorob'evy Gory, MGU, 119234 \\ Moscow, Russian Federation
}

\begin{abstract}
We study how the presence of the middle class in the sense of Gevorgyan-Malykhin affects the value of income inequality measures including the Gini coefficient $J$ and the Hoover index H. It is proved that in the presence of the middle class (1) $J \leq \frac{1}{2}-\frac{L^{\prime}(0)}{2}$ (where $L$ is the Lorenz function), (2) $H \leq \frac{1}{2}$, (3) the longest vertical distance between the diagonal and the Lorenz curve (which is equal to $\mathrm{H}$ ) is attained at $z_{0}<\frac{3}{4}$. A tight upper bound for P90/P10 ratio is found assuming $L^{\prime}(0)>0$. Tight upper and lower bounds for the differential deviation in terms of the Gini coefficient are found as well.
\end{abstract}

\section{Introduction}

Roughly speaking the middle class is some middle part of a society in social or socioeconomic hierarchy. There is no precise well-established definition of the middle class but most often it is defined in terms of income. According to Ravallion [1], "typically, being "middle class" is defined as having an income within some interval that includes the median and the interval has typically been symmetric in the income space around the median". The middle class is believed to play an important stabilizing role in modern society [2]. In order to do so effectively, obviously, it must constitute a sizable portion of a society and possess a sizable portion of its income.

Recall that the Lorenz function $L(z)$ is the fraction of the total income or wealth, owned by the poorest fraction $z$ of the population.

\footnotetext{
*Corresponding author: pavlov_oi@rudn.ru
} 


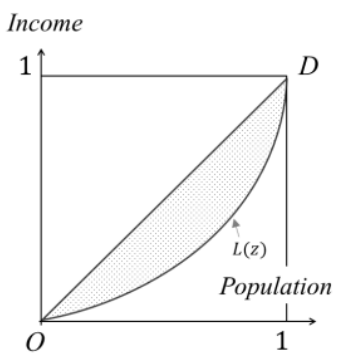

Fig. 1. The Lorenz curve $L(z)$. The doubled area of the shaded region is the Gini coefficient.

How much wealth may belong to the middle part (i.e. the second and third quartiles) of the population?

In case of the perfect equality $L(z)=z$ so that $L\left(\frac{3}{4}\right)-L\left(\frac{1}{4}\right)=\frac{3}{4}-\frac{1}{4}=\frac{1}{2}$. In the example below the richest $25 \%$ own more than $50 \%$ of wealth so $L\left(\frac{3}{4}\right)<\frac{1}{2}$, hence $L\left(\frac{3}{4}\right)-L\left(\frac{1}{4}\right)<\frac{1}{2}$.

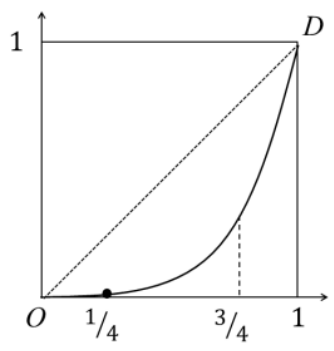

Fig. 2. $L\left(\frac{3}{4}\right)<\frac{1}{2}$ implies the absence of the middle class.

Gevorgyan and Malykhin [3] gave the following mathematical definition: there is the middle class if $L\left(\frac{3}{4}\right)-L\left(\frac{1}{4}\right) \geq \frac{1}{2}$. Equivalently, there is the middle class if the middle part of the population (if ordered by wealth) possesses at least one-half the total wealth. Having the middle class prohibits the richest quarter of the society to have over a half of the total wealth. For example, if $L(z)=z^{3}$, then $L\left(\frac{3}{4}\right)=\left(\frac{3}{4}\right)^{3}=\frac{27}{64}<\frac{1}{2}$, hence there is no middle class in a such a society. Similarly, there is no middle class in a society with the Lorenz curve from Figure 2, but there is a middle class in case of the perfect equality. The authors proved in [4] that in the class of two-segment polygonal Lorenz curves (with the vertices $(0 ; 0),(a ; b)$, and $(1 ; 1))$ there is the middle class if and only if $a \leq \frac{1}{2}$.

It is clear that the presence of the middle class does influence the distribution of wealth in a society. In order to measure such an influence numerically we shall consider several inequality indices (which can be interchangeably applied both to income and savings). The most popular one, the Gini coefficient, was defined in [5], also see [6]. Geometrically it can be described as the fraction of the area between the Lorenz curve and the diagonal AD (which is shaded in Figure 1) relative to the area of the triangle under that diagonal. The closer is the Lorenz curve to the diagonal, the smaller is the Gini index. Given a Lorenz curve L, we denote the correspondent Gini coefficient by $J(L)$ or simply by $J$.

The Hoover index [7] is the largest deviation of $L(z)$ from the identity, that is, the maximal value of $z-L(z)$. The Hoover index is also known as the Pietra index and the Schutz index; it is also called the Robin Hood index because it equals to the amount of wealth needed to be redistributed from the rich to the poor in order to attain the perfect equality. We also consider the P90/P10 ratio which is the ratio of the upper bound value of income of the 
$90 \%$ of people with highest income (i.e. of the ninth decile) to that of the $10 \%$. Yet another inequality measure - differential deviation - was introduced in [8] as $\int_{0}^{1}\left(L^{\prime}(z)-1\right)^{2} d z$.

The main research question considered in this paper is: How can the presence of a middle class affect the value of the Gini coefficient and other income inequality measures? Throughout the paper we assume $L(z)$ to be continuous.

\section{Results and discussion}

\subsection{The Gini coefficient}

Gevorgyan and Malykhin proved that if there is the middle class, then $J \leq \frac{1}{2}$. But the maximal value $J=\frac{1}{2}$ is attained at $L(z)$ which is equal to zero on $[0 ; 0.5]$ (in such a society the poorest one half of the population one does not have any income whatsoever). It is unlikely that such a distribution of wealth is possible in a modern society! So we will assume that every initial segment of the population does have some income. As $L^{\prime}(z)$ is proportional to the income of person/household corresponding to $z$, it is feasible to consider the case $L^{\prime}(0)>0$.

What are the possible values of the Gini coefficient given $L^{\prime}(0)>0$ in the presence of the middle class? We answer this question in the following Theorem 1:

Theorem 1. If there is the middle class, then $J \leq \frac{1}{2}-\frac{L^{\prime}(0)}{2}$.

Proof of theorem 1. Denote $L^{\prime}(0)$ by $k$. Since $L(z)$ is concave up (convex), the Lorenz curve lies on or above the line $y=k x$. Since $L\left(\frac{3}{4}\right)-L\left(\frac{1}{4}\right) \geq \frac{1}{2}$ and $L\left(\frac{1}{4}\right) \geq \frac{k}{4}$, we have that $L\left(\frac{3}{4}\right) \geq \frac{1}{2}+\frac{k}{4}$. Due to convexity, the Lorenz curve is on or above some straight line $l_{1}$ which goes through the point $\left(\frac{3}{4}, L\left(\frac{3}{4}\right)\right) \cdot\left(l_{1}\right.$ coincides with the tangent line to the Lorenz curve at $\left(\frac{3}{4}, L\left(\frac{3}{4}\right)\right)$ if the latter exists.) By shifting $l_{1}$ by $L\left(\frac{3}{4}\right)-\left(\frac{1}{2}+\frac{k}{4}\right)$ units down, we obtain a straight line $l_{2}$ which goes through the point $\left(\frac{3}{4}, \frac{1}{2}+\frac{k}{4}\right)$ and such that the Lorenz curve is on or above $l_{2}$. Also, the vertex $\mathrm{D}$ is on or above $l_{2}$ since the Lorenz curve is convex. Then the upper envelope of the lines $y=k x$ and $l_{2}$ is a two-segment polygonal chain OAB (see Figure $3)$ which contains the points $(0 ; 0)$ and $\left(\frac{3}{4} ; \frac{1}{2}\right)$ such that no point of the Lorenz curve lies below it. Hence the area between the diagonal AD and the Lorenz curve is not greater than the area of the quadrilateral OABD. This means that $J(L)$ is not greater than twice the area of OABD which is further denoted by $\mathrm{S}_{\mathrm{OABD}}$.

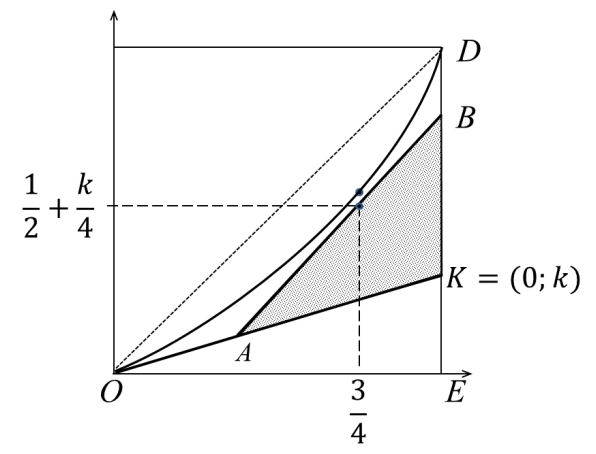

Fig. 3. The line $l_{2}$ (not shown) goes through the points $\mathrm{A},\left(\frac{3}{4}, \frac{1}{2}+\frac{k}{4}\right)$, and B. 
Denote the point $(1 ; k)$ which is the intersection of the line $y=k x$ and the segment ED by K. Since $S_{O A B D}=\frac{1}{2}-S_{O E K}-S_{A B K}$ and $S_{O E K}$ is equal to $\frac{k}{2}$, it is enough to estimate $S_{A B K}$. Denote the point $\left(\frac{3}{4}, \frac{1}{2}+\frac{k}{4}\right)$ by $K^{\prime}$. Let segments $K^{\prime} B^{\prime}$ and $K^{\prime} A^{\prime}$ be parallel to $A K$ and $B K$ respectively, see Figure 4. The distance between the lines $K^{\prime} A^{\prime}$ and $B^{\prime} K$ is equal to $\frac{1}{4}$ since both are vertical and the second coordinates of $K^{\prime}$ and $B$ are $\frac{3}{4}$ and 1 respectively. The second coordinate of $A^{\prime}$ is equal to $\frac{3}{4} k$ since $A^{\prime}$ is on the line $y=k x$ and the first coordinate of $A^{\prime}$ is $\frac{3}{4}$ (the same as that of $K^{\prime}$ ). Therefore $\left|K^{\prime} A^{\prime}\right|=\frac{1}{2}+\frac{k}{4}-3 \frac{k}{4}=\frac{1}{2}-\frac{k}{2}$. This means that $S_{A^{\prime} K^{\prime} B^{\prime} K}=\frac{1}{4}\left(\frac{1}{2}-\frac{k}{2}\right)=\frac{1}{8}-\frac{k}{8}$.

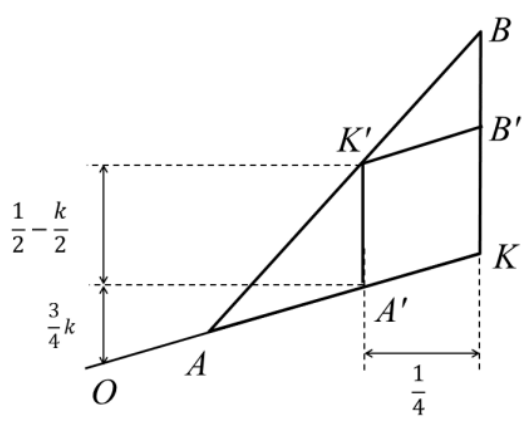

Fig. 4. Calculating the area of the parallelogram $A^{\prime} K^{\prime} B ' K$.

It is proved in [9] that if a triangle $K A B$ is cut off an angle $K$ by a line, which goes through an interior point $K^{\prime}$ of the angle, then this triangle has the minimum area if $A K^{\prime}=K^{\prime} B$. In order to consider this case, we need to check whether $B$ belongs to the segment $E D$. We have that $B B^{\prime}=B^{\prime} K$ since $K^{\prime} B=A K^{\prime}$ and $K^{\prime} B^{\prime} \| A K .\left|B^{\prime} K\right|=\left|K^{\prime} A^{\prime}\right|=\frac{1}{2}-\frac{k}{2}$, therefore $|B E|=$ $B B^{\prime}+B^{\prime} K+K E=\left(\frac{1}{2}-\frac{k}{2}\right)+\left(\frac{1}{2}-\frac{k}{2}\right)+k=1$. This means that $B$ coincides with $D$, hence $S_{O A B D}=\frac{1}{2}-S_{O E K}-S_{A B K}$ in the considered case indeed. Both triangles $K^{\prime} B B^{\prime}$ and $A K^{\prime} A^{\prime}$ are similar to the triangle $A B K$ and are in proportion $\frac{1}{2}$ to it. Therefore, $S_{K^{\prime} B B^{\prime}}=S_{A K A^{\prime}}=$ $\frac{1}{4} S_{A B K}$. This implies that the area of the parallelogram $A^{\prime} K^{\prime} B^{\prime} K$ is one half of $S_{A B K}$, so $S_{A B K} \geq$ $2 S_{A^{\prime} K^{\prime} B^{\prime} K}=2\left(\frac{1}{8}-\frac{k}{8}\right)=\frac{1}{4}-\frac{k}{4}$. So $S_{O A B D}=\frac{1}{2}-S_{O E K}-S_{A B K} \leq \frac{1}{2}-\frac{1}{2} k-\left(\frac{1}{4}-\frac{k}{4}\right)=\frac{1}{4}-\frac{k}{4}$. Thus $J(L) \leq 2 S_{O A B D} \leq 2\left(\frac{1}{4}-\frac{k}{4}\right)=\frac{1}{2}-\frac{k}{2}=\frac{1}{2}-\frac{L^{\prime}(0)}{2}$. The proof is complete.

The estimate for the Gini coefficient given by Theorem 1 is sharp. Indeed, for the twosegment polygonal Lorenz curve $L_{k}$ with vertices $(0 ; 0),\left(\frac{1}{2} ; \frac{k}{2}\right)$, and $(1 ; 1), L^{\prime}(0)=k$ because the first segment is a part of the line $y=k x$. The estimate for Gini coefficient, given by the theorem, is $\frac{1}{2}-\frac{k}{2}$. The actual value of $J\left(L_{k}\right)$ is equal to the difference of the coordinates of the midpoint $\left(\frac{1}{2} ; \frac{k}{2}\right)$ (see [10]), which equals $\frac{1}{2}-\frac{k}{2}$ too. In the worst case $L^{\prime}(0)=0$ Theorem 1 reduces to the theorem of Gevorgyan and Malykhin.

Note that without a requirement for the middle class the Gini coefficient may reach 1 $L^{\prime}(0)$ (this value is attained at the two-segment polygonal Lorenz curve with vertices $(0 ; 0)$, $(1 ; k)$, and $(1 ; 1)$. Therefore, the presence of the middle class cuts the Gini coefficient in half. 


\subsection{The Hoover index}

Let $z_{0}$ be such that $z_{0}-L\left(z_{0}\right)$ is maximal among $z-L(z)$ for all $0 \leq z \leq 1$. (In other words, $z_{0}$ corresponds to the person/household having the average income. If $z_{0}$ is unique, then every person from $\left[0 ; z_{0}\right)$ has a below average income, while very person from $\left(z_{0} ; 1\right]$ has an above average income.) In general, $z_{0}$ can be any single number from the interval $(0 ; 1)$ (this observation can easily be seen from the fact that $\left(z_{0} ; L\left(z_{0}\right)\right)$ is the point of the Lorenz curve which is the most remote from the diagonal $\mathrm{AD}$ ), or it can be an interval of numbers. The following Figure 5 shows three Lorenz curves corresponding to the same value of the Hoover index (and the same value of the Gini coefficient as well because for a two-segment polygonal Lorenz curves the Hoover index is equal to the Gini coefficient), but attaining their average income at different $z_{0}$.

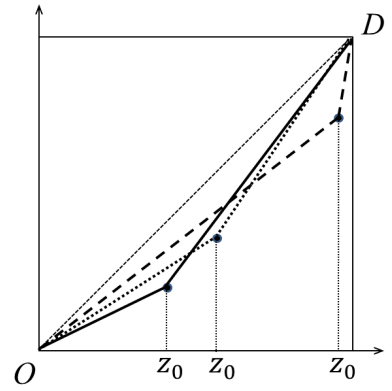

Fig. 5. Three Lorenz curves with the same Hoover index and Gini coefficient but with average income values at various $z_{0}$.

For simplicity, we assume that in the following theorem the Lorenz function is differentiable on $(0 ; 1)$.

Theorem 2. If there is the middle class, then

a. There is $z_{0}<\frac{3}{4}$.

b. $H \leq \frac{1}{2}$.

Proof of theorem 2.

a. According to the mean value theorem there exists $z^{\prime} \in\left[\frac{1}{4} ; \frac{3}{4}\right]$ such that $L^{\prime}\left(z^{\prime}\right)=\left(L\left(\frac{3}{4}\right)-L\left(\frac{1}{4}\right)\right):\left(\frac{3}{4}-\frac{1}{4}\right)$. Then $L^{\prime}\left(z^{\prime}\right) \geq 1$ since the denominator in the latter formula is equal to $\frac{1}{2}$ and because $L\left(\frac{3}{4}\right)-L\left(\frac{1}{4}\right) \geq \frac{1}{2}$ in the presence of the middle class. $L^{\prime}(z)$ is a nondecreasing function because $L(z)$ is concave up. Hence the set of all $z \in[0 ; 1]$ such that $L^{\prime}\left(z^{\prime}\right)<1$ is an initial interval of $[0 ; 1]$ which does not contain $z^{\prime} \leq \frac{3}{4}$ by the above argument. Clearly the right endpoint of this segment is the required $z_{0}$ such that $z_{0} \leq z^{\prime} \leq \frac{3}{4}$.

b. The presence of the middle class implies that $L\left(\frac{3}{4}\right) \geq \frac{1}{2}$. Then since $z_{0} \leq \frac{3}{4}$ by item a. and due to convexity of $L(z)$, the point $\left(\frac{3}{4}, \frac{1}{2}\right)$ should be on or below the segment with the endpoints $P=\left(z_{0} ; L\left(z_{0}\right)\right)$ and $(1 ; 1)$. This means that $P$ is on or above the line which goes through the points $\left(\frac{3}{4}, \frac{1}{2}\right)$ and $(1 ; 1)$ (see Figure 6$)$. 


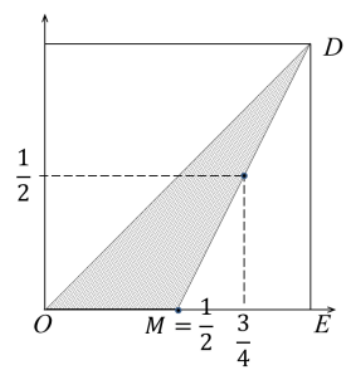

Fig. 6. Possible position of $P$.

Therefore, $P$ belongs to the triangle $O D M$ (to its part which is to the left of the vertical line $z=\frac{3}{4}$ ). However, the longest vertical segment lying in this triangle contains $M$ and is of length $\frac{1}{2}$. So $H(L) \leq \frac{1}{2}$. Theorem 2 is proved.

Note that the set of all $z_{0}$ is not necessarily bounded by $z=\frac{3}{4}$. For example, in case of the perfect equality $(L(z) \equiv z)$ this set is exactly $[0 ; 1]$. Theorem 2 states that this set, if not singleton, necessarily contains $z_{0}<\frac{3}{4}$.

The maximal possible value of the Hoover index in the presence of the middle class, which is $\frac{1}{2}$, is attained at the two-segment polygonal Lorenz curve with vertices $(0 ; 0),\left(\frac{1}{2} ; 0\right)$, and $(1 ; 1)$.

\subsection{The P90/P10 ratio}

Since $L^{\prime}(z)$ is proportional to income/prosperity of a person, the P90/P10 ratio which is the ratio of the ninth decile to tenth decile is also equal to $L^{\prime}(0.9) / L^{\prime}(0.1)$ (in this section we assume $L(z)$ to be differentiable on $(0 ; 1))$. Clearly the $\mathrm{P} 90 / \mathrm{P} 10$ ratio $\geq 1$; it is well-known that the P90/P10 ratio can be any number $\geq 1$. [11] noted that the presence of the middle class does not affect the P90/P10 ratio, i.e. it can still be any number $\geq 1$.

Theorem 3. Let $L^{\prime}(0)>0$.

a. $P 90 / P 10 \leq \frac{10-9 L^{\prime}(0)}{L^{\prime}(0)}$.

b. If there is the middle class, then $P 90 / P 10 \leq \frac{3.5-2.5 L^{\prime}(0)}{L^{\prime}(0)}$.

Proof of theorem 3. Denote $L^{\prime}(0)$ by $k$. Throughout the proof we shall use the mean value theorem according to which $L(b)-L(a)=L^{\prime}(t)(b-a)$ for some $t \in(a, b)$. Then $L^{\prime}(t) \geq 0$ implies that $L(b)-L(a) \geq L^{\prime}(t)(b-a) . L^{\prime}(z)$ is a non-decreasing function because $L(z)$ is concave up, therefore $L^{\prime}(t) \geq L^{\prime}(a)$ and

$$
L(b)-L(a) \geq L^{\prime}(a)(b-a)
$$

whenever $\mathrm{b} \geq \mathrm{a}$. Now the proof splits in two parts.

a. It then follows from (1) that $L(0.9) \geq L^{\prime}(0) \cdot 0.9=0.9 \mathrm{k}$. Due to convexity of $L(z)$, $L^{\prime}(0.9)$ does not exceed the slope of the secant line which goes through points $(0.9 ; L(0.9))$ and $(1 ; 1)$. This slope is equal to

$\frac{1-L(0.9)}{0.1} \leq \frac{1-0.9 k}{0.1}=10-9 k$.

Hence $L^{\prime}(0.9) \leq 10-9 k$. Now the inequality $L^{\prime}(0.1) \geq k$ implies that $P 90 / P 10 \leq$ $\frac{10-9 k}{k}=\frac{10-9 L^{\prime}(0)}{L^{\prime}(0)}$ as required. 
b. Similarly to the proof of the item a, we have that $L(0.25) \geq L^{\prime}(0) \cdot 0.25=0.25 k$.

$L(0.75)-L(0.25) \geq \frac{1}{2}$ due to the presence of the middle class.

$L^{\prime}(0.75)$ is not less than the slope of the secant line which goes through points $(0.25 ; L(0.25))$ and $(0.75 ; L(0.75))$, thus $L^{\prime}(0.75) \geq 1$.Consequently, $L(0.9)-$ $L(0.75) \geq L^{\prime}(0.75) \cdot 0.15 \geq 0.15$. Therefore,

$L(0.9)=L(0.25)+(L(0.75)-L(0.25))+(L(0.75)-L(0.25)) \geq 0.25 k+0.5+$ $0.15=0.25 k+0.65$.

As in the previous item, $L^{\prime}(0.9)$ does not exceed the slope of the secant line which goes through points $(0.9 ; L(0.9))$ and $(1 ; 1)$, which is equal to

$\frac{1-L(0.9)}{0.1} \leq \frac{1-0.25 k-0.65}{0.1}=\frac{0.35-0.25 k}{0.1}=3.5-2.5 k$.

Finally, $P 90 / P 10 \leq \frac{3.5-2.5 k}{k}=\frac{3.5-2.5 L^{\prime}(0)}{L^{\prime}(0)}$ as required.

In both items of Theorem 3 the upper estimates for the P90/P10 ratio are of the form $a+$ $\frac{b}{L^{\prime}(0)}$. We note that neither $a$, nor $b$ can be decreased (independently of the other). Indeed, consider the following Lorenz curves: the two-segment polygonal Lorenz curve $L_{1}$ with vertices $(0 ; 0),(1 ; k)$, and $(1 ; 1)$ and at the three-segment polygonal Lorenz curve $L_{2}$ with vertices $(0 ; 0),(0.25 ; 0.25 k),(0.9 ; 0.25 k+0.65)$, and $(1 ; 1)$. The former one can be modified into a smooth curve if we replace its part to the right of the line $z=0.95$ with a piece of parabola which connects the points $(0.95 ; 0.95 k)$ and $(1 ; 1)$ and has the necessary derivative (namely, $k$ ) at $z=0.95$. The P90/P10 ratio for this curve is exactly $\frac{10-9 k}{k}=$ $\frac{10-9 L^{\prime}(0)}{L^{\prime}(0)} . L_{2}$ can also be modified into a smooth curve. For this, shift the left endpoint of the third segment up by a small $\varepsilon>0$ to obtain the point $(0.9 ; 0.25 k+0.65+\varepsilon)$ and connect the second segment with the old first segment and the new third segment by smooth curves (such as an arc of a parabola or a circle). The P90/P10 ratio for the new curve is slightly less than $\frac{3.5-2.5 k}{k}=\frac{3.5-2.5 L^{\prime}(0)}{L^{\prime}(0)}$ and it gets as close to $\frac{3.5-2.5 k}{k}$ as desired as $\varepsilon$ is getting closer to zero.

\subsection{The differential deviation}

This index was proposed in [8] as $\int_{0}^{1}\left(L^{\prime}(z)-1\right)^{2} d z$. Because defined in terms of the Lorenz function, this index does satisfy desirable properties of income inequality measures (see [12]). Also, it was shown [8] to be coherent with the natural partial order on the set of Lorenz curves. Throughout the rest of the paper the differential deviation of a society with the Gini coefficient $J$ will be denoted by $D(J)$ or simply by $D$. We will use the following fact from [4]: there is the middle class for a two-segment polygonal Lorenz curve if and only if its middle vertex is on or to the left of the line $z=\frac{1}{2}$.

Theorem 4. For a two-segment polygonal Lorenz curve:

a. If $J \leq \frac{1}{2}$, then $4 J^{2}+1 \leq D(J) \leq \frac{1}{1-J}$.

b. If $J>\frac{1}{2}$, then $D(J) \geq \frac{1}{1-J}$ and $D(J)$ can be indefinitely large.

Proof of theorem 4. a. It was proved in [13] that $D(J) \geq 4 J^{2}+1$ for a two-segment polygonal Lorenz curve whenever $J \leq \frac{1}{2}$.

As derived in [13], $D(J)=J^{2}\left(\frac{1}{z_{0}}+\frac{1}{1-z_{0}}\right)+1$, where $\left(z_{0}, z_{0}-J\right)$ is the middle vertex of the two-segment Lorenz curve. The presence of the middle class implies that $z_{0} \leq \frac{1}{2}$; the fact 
that the vertex $\left(z_{0}, z_{0}-J\right)$ is in the unit square means that $z_{0}-J \geq 0$. Hence $J \leq z_{0} \leq \frac{1}{2}$. But it is known that given segment $I \subset\left[0 ; \frac{1}{2}\right]$, the largest value of $\frac{1}{z}+\frac{1}{1-z}$ on $I$ is attained at that endpoint of $I$ which is closer to $z=0$, and for the segment $\left[J ; \frac{1}{2}\right]$ this endpoint is $z=J$. Consequently,

$$
D(J) \leq J^{2}\left(\frac{1}{J}+\frac{1}{1-J}\right)+1=J^{2}\left(\frac{1}{J(1-J)}\right)+1=\frac{J}{1-J}+1=\frac{1}{1-J}
$$

b. $D(J) \geq \frac{1}{1-J}$ whenever $J>\frac{1}{2}$ according to [13]. Fix $J$. If $z_{0}$ is sufficiently close to 1 , then $\frac{1}{1-z_{0}}$ can be indefinitely large and so will $D(J)=J^{2}\left(\frac{1}{z_{0}}+\frac{1}{1-z_{0}}\right)+1$.

Theorem 4 is proved.

Corollary 1. If there is the middle class, then $4 J^{2}+1 \leq D(J) \leq \frac{1}{1-J}$.

Proof of Corollary 1 . If there is the middle class, then $J \leq \frac{1}{2}$ according to GevorgyanMalykhin theorem. The minimum value $4 J^{2}+1$ is attained at the curve with vertices $(0 ; 0)$, $\left(\frac{1}{2} ; \frac{1}{2}-J\right)$, and $(1 ; 1)$ (see [13]) for which there is the middle class according to the fact from [4]. The two-segment polygonal Lorenz curve with vertices $(0 ; 0),(J ; 0)$, and $(1 ; 1)$ at which the maximal value $\frac{1}{1-J}$ is attained has the middle class due to the same fact.

\subsection{Question}

What are the possible values of $D(J)$ in the presence of the middle class (if the Lorenz curve is not necessarily a two-segment polygonal chain)?

\subsection{Further discussion}

The study of the middle class in the sense of Gevorgyan-Malykhin can be continued in several directions. One direction is to relax the concept and allow for the difference $L\left(\frac{3}{4}\right)-$ $L\left(\frac{1}{4}\right)$ to be less than $\frac{1}{2}$. In [4] the authors gave a definition of an $\varepsilon$-middle class by replacing the inequality $L\left(\frac{3}{4}\right)-L\left(\frac{1}{4}\right) \geq \frac{1}{2}$ with $L\left(\frac{3}{4}\right)-L\left(\frac{1}{4}\right)>\frac{1}{2}-\varepsilon$. This definition is more stable than the original one in the sense that if a given Lorenz curve $L$ satisfies the inequality $L\left(\frac{3}{4}\right)-$ $L\left(\frac{1}{4}\right)>\frac{1}{2}-\varepsilon$, then a sufficiently close $\tilde{L}$ will also satisfy it (for example, in the topology of uniform convergence).

It is possible to quantify to which extent the middle class is absent. We called in [14] the difference $\frac{1}{2}-\left(L\left(\frac{3}{4}\right)-L\left(\frac{1}{4}\right)\right)$ the middle-class deficit and proved that $L\left(\frac{3}{4}\right)-L\left(\frac{1}{4}\right) \geq \frac{1}{2}-$ $\frac{2}{3} J$ for every Lorenz curve $L$. In other words, the middle-class deficit never exceeds $\frac{2}{3} J$.

Also, it is possible to stipulate one or another degree of homogeneity of the middle class itself. For example, Gevorgyan and Malykhin [3] suggested to consider the P75/P25 ratio and they gave an upper estimate for the Gini coefficient provided the middle class is present and there is a perfect equality within the second and third quartiles of the population (the latter condition implies that the Lorenz curve is linear between $z=0.25$ and $z=0.75$ ). 


\section{Conclusions}

We established that the presence of the middle class in the sense of Gevorgyan-Malykhin strongly influences various income inequality measures. The values of the Gini coefficient and the Hoover index are cut in half. The upper bound for the P90/P10 ratio (provided $L^{\prime}(0)>0$ ) is reduced by almost three times. On the contrary, both the upper and lower bounds on the differential deviation in terms of the Gini coefficient are attained on the middle-class Lorenz curves whenever $J \leq \frac{1}{2}$.

\section{References}

1. M. Ravallion, World Development, 38 (2009)

2. N. Vi. Kuznetsova, Ar. Y. Tupitsyn, Asian Social Science, 10 (2014)

3. P. S. Gevorgyan, V. I. Malykhin, Labor and Social Relations, 12, 90 (2010)

4. O. I. Pavlov, O. Yu. Pavlova, Innovations in life, 4, 153 (2016)

5. C. Gini, Bologna (1912)

6. L. Ceriani, P. Verme, J. Econ, Inequal, 10 (2012)

7. E. Hoover, The Review of Economics and Statistics, 18 (1936)

8. O. I. Pavlov, O. Yu. Pavlova, Management of economic systems, 12 (2017) http://uecs.ru

9. Line through a Point in an Angle, https://www.cut-the-knot.org

10. A. Frank, Farris, The American Mathematical Monthly, 117 (2010)

11. A. N. Astashenko, V. I. Malykhin, Indicators of social differentiation (LAP LAMBERT Academic Publishing, 2012)

12. Hugh Dalton, The Economic Journal, 30 (1920)

13. O. I. Pavlov, O. Yu. Pavlova, Russian Economics online-journal, 4 (2018) https://readera.org

14. O. I. Pavlov, O. Yu. Pavlova, Management of economic systems, 12 (2016) http://uecs.ru 